\title{
Realization and Application of a Sub-linear Time Complexity Correlation Algorithm to a Passive Radar System
}

\author{
Kangning Zhao ${ }^{1, a}$, Peizhong $\mathrm{Lu}^{2, \mathrm{~b}}$, Yan Zou ${ }^{3, \mathrm{c}}$, Jinsong Wang ${ }^{4, \mathrm{~d}}$, \\ Lin Ling ${ }^{5, e}$ and Kai Cao ${ }^{6, f}$
}

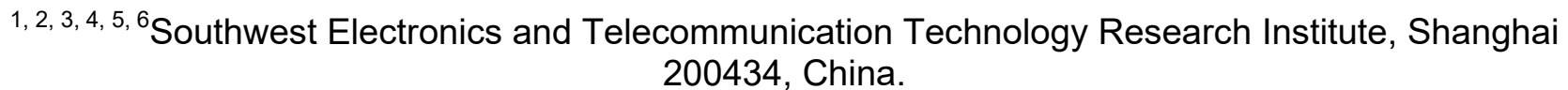

akn_zhao@163.com, bpzlu@fudan.edu.cn, cyanzou@fudan.edu.cn, djinsong.wang@126.com, 'lynn-00@163.com, ck1988@mail.ustc.edu.cn

Keywords: sub-linear time, FFT, folding, passive radar

\begin{abstract}
Correlation calculation plays a very significant role in many scientific fields such as signal processing and string matching. Finding a faster and reliable correlation calculation is very helpful to improve the performance of the system. This paper applies a sub-linear time complexity correlation algorithm proposed in [1] from a MIT research group to a passive radar system to realize the real-time track of aircrafts, which makes the system more effective. Also this paper makes experimental analysis to this algorithm from the parameter properties, time complexity and anti-error, which proves that the time complexity of the algorithm is much lower than the traditional FFT algorithm, which is a very big advantage.
\end{abstract}

\section{Introduction}

Correlation calculation plays a very significant role in many scientific fields, such as signal processing, cryptography and string matching, which means an excellent correlation algorithm has a great value in scientific application. With the development of communication technology, the amount of correlation calculation grows rapidly which the request of real-time performance is higher in the computational system, which makes finding a faster and reliable correlation system a very important research subject. Especially it has a great value in passive radar system to track and find the target to achieve the real-time surveillance.

We all know that traditional correlation algorithm based on FFT can reduce the time complexity of correlation to $\mathrm{O}(\mathrm{N} \log \mathrm{N})$, which improves the computational efficiency. But it is still a linear complexity algorithm and it can't meet the demand of real-time calculation in many applications.

In recent years, scholars and researchers have done a lot of research works on this issue in various kinds of background. A new sparse Fast Fourier transform algorithm is proposed in [2] by Professor H. Hassanieh and his team in MIT, which reduces the time complexity of FFT to $\mathrm{O}\left(\mathrm{N}(\log \mathrm{N})^{1 / 2}\right)$, which is sparse Fast Fourier Transform. They applies this algorithm into GPS signal synchronization in [3] and other works, which proves the excellent performance of the algorithm.

However, the time complexity of the correlation calculation is still linear, which still can't meet our request. A sub-linear correlation algorithm is proposed in [1] by Andoni et al., which further reduces the time complexity to $\mathrm{O}\left((\mathrm{N} \log \mathrm{N})^{2 / 3}\right)$. It has a great value in various applications of scientific and technological fields.

This paper applies the algorithm to a passive radar system and improve it, which indicates the good performance of the algorithm to track the target of aircraft in real time.

\section{Brief Introduction of the Sub-linear Algorithm [1]}

The core of the algorithm proposed in [1] is "folding". Assuming that we have to do a calculation of two data code vectors $\mathbf{c}$ and $\mathbf{x}$, the lengths of which are both $\mathrm{N}$, and we have that:

$$
\mathbf{x}=\mathbf{c}^{(\tau)}+\mathbf{g} \text {. }
$$

That is, $\mathbf{x}$ is the $\tau$-shift of $\mathbf{c}$ together with a white noise code vector $\mathbf{g}$. 
The meaning of the folding is: we separate the data code $\mathbf{c}$ into $\mathrm{p}$ groups, for each group we add the $\mathrm{N} / \mathrm{p}$ points of data together and use the sum to represent the group. The $\mathrm{p}$ sums form a new folding code vector $\mathbf{c}(\mathrm{p})$, that is similar with $\mathbf{x}(\mathrm{p})$. They have a property: if $\mathbf{x}$ matches $\mathbf{c}^{(\tau)}$ then $\mathbf{x}(\mathrm{p})$ matches $\mathbf{c}(\mathrm{p})^{\left(\mathrm{t}^{\prime}\right)}$ and $\mathrm{t}^{\prime} \equiv \tau \bmod (\mathrm{N} / \mathrm{p})$. So we can find the shift $\mathrm{t}^{\prime}$ by doing correlation $\mathbf{x}(\mathrm{p})$ and $\mathbf{c}(p)$. To ensure the time complexity of the whole algorithm is sub-linear, we just use part of the points of $\mathbf{x}(\mathrm{p})$ and $\mathbf{c}(\mathrm{p})$ to do the correlation. After that, we can search the final result among the $p$ possible shift $t_{j}=t^{\prime}+j \cdot N / p, j \in\{0,1,2, \ldots, p-1\}$ by doing correlation of $\mathbf{x}$ and $\mathbf{c}$, using part of points of them. The whole math description of the algorithm is that:

We have $\mathbf{x}$ and $\mathbf{c}, \mathbf{x}(\mathrm{p})$ and $\mathbf{c}(\mathrm{p})$, let $l=\mathrm{N} / \mathrm{p}, l$ is an integer. For each of the element of c(p), we have that folding sum:

$$
\mathrm{c}(\mathrm{p})_{\mathrm{i}}=\sum_{\mathrm{j}=0}^{\mathrm{p}-1} \mathrm{c}_{\mathrm{i}+\mathrm{jn} / \mathrm{p}} \text {. }
$$

We have the set $X \subset\{0,1, \ldots, N-1\}$, and we define $\left.\mathbf{c}\right|_{X}$ as the projection of the data $\mathbf{c}$ in the set $X$ (e.g. If $X=\{5,7,15\}$, then $\left.\mathbf{c}\right|_{X}=\left[c_{5}, c_{7}, c_{15}\right]$ ), and it can be described as the following:

1) Calculate $\left.\mathbf{x}(\mathrm{p})\right|_{X}$, and set $X=\{0, \ldots, \sqrt{l \cdot \log \mathrm{N}}-1\}$.

2) Calculate $\left.\mathbf{c}(\mathrm{p})\right|_{\mathrm{Y}}$, and we have the set:

$$
\mathrm{Y}=\mathrm{Y}_{0} \cup \ldots \cup \mathrm{Y}_{\sqrt{l}-1}, \mathrm{Y}_{\mathrm{i}}=\{\mathrm{i} \sqrt{l}, \mathrm{i} \sqrt{l}+1, \ldots, \mathrm{i} \sqrt{l}+\mathrm{s}-1\}, \mathrm{s}=\mathrm{O}(\sqrt{\log \mathrm{N}}) .
$$

3) For every possible shift $\mathrm{t} \subset\{0,1,2, \ldots, l-1\}$ in the first step, we have:

$$
d_{t}=\sum_{i \in X \cap\{Y-t\}} c(p)_{i}^{(t)} \cdot x(p)_{i}=\sum_{i \in X \cap\{Y-t\}} c(p)_{i+t} \cdot x(p)_{i} .
$$

And we can see that $\mathrm{X} \subset\{\mathrm{Y}-\mathrm{t}\}$ is the set of coordinates of points selected to do correlation.

4) We can get the first shift:

$$
t^{\prime}=\underset{t}{\operatorname{argmax}} d_{t} \text {. }
$$

5) Get the final shift:

$$
\tau=\underset{\mathrm{t}_{\mathrm{j}}=\mathrm{t}^{+}+\mathrm{j}: \mathrm{N} / \mathrm{p}}{\operatorname{argmax}} \sum_{\mathrm{i} \in \mathrm{S}} \mathrm{c}_{\mathrm{i}}^{\left(\mathrm{t}_{\mathrm{j}}\right)} \cdot \mathrm{x}_{\mathrm{i}}, \mathrm{S}=\{0,1, \ldots, \mathrm{O}(\log \mathrm{N})\} .
$$

It is pointed in [1] that when $\mathrm{p}=(\mathrm{N} \log \mathrm{N})^{1 / 3}$, the time complexity of the algorithm is $\mathrm{O}\left((\mathrm{N} \log \mathrm{N})^{2 / 3}\right)$, which is sub-linear.

\section{Experimental Analysis of the Sub-linear Algorithm}

We have done a lot of experiments of this algorithm proposed in [1] and experimental analysis is presented in this paper in the following three aspects:

1. The influence of folding factor $p$ on the running time of the algorithm.

2. The comparison of the running time with the FFT algorithm.

3. The anti-error analysis of the algorithm.

For the influence of folding factor $p$ on the running time of the algorithm, we just pick the vector length of $\mathrm{N}^{20}$, and for the different value of $\mathrm{p}$, we have the curve of the running time in the following Fig. 1: 


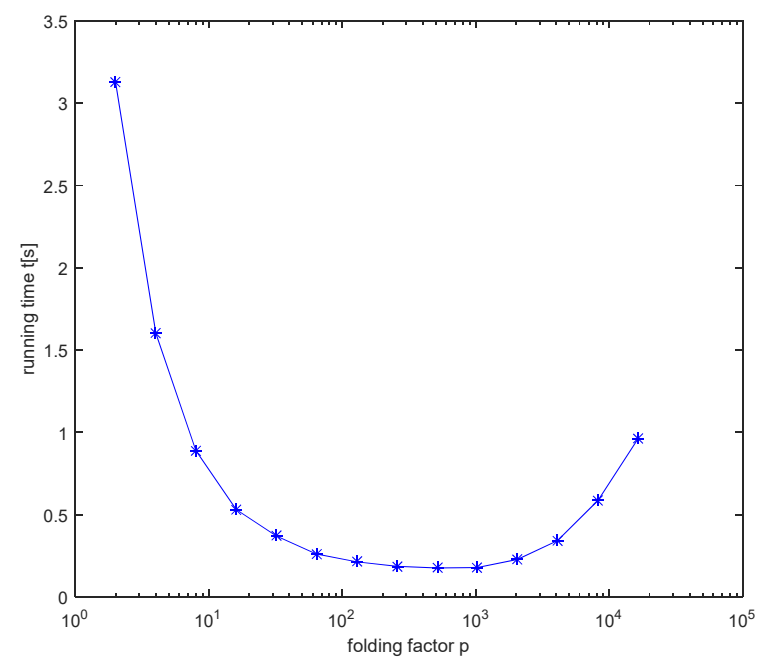

Fig. 1 The curve of the running time $t$ with the folding factor $p$ changing

From Fig. 1, we can see that the running time of the algorithm has a minimum value, approximately $0.2 \mathrm{~s}$ when $\mathrm{p}$ is near to $2^{8}$, and we can see that when $\mathrm{N}=2^{20}$, then $(\mathrm{N} \log \mathrm{N})^{1 / 3}=$ 275.77, which is close to $2^{20}$ and meets the theoretical analysis in [1].

For the comparison of the running time with the FFT algorithm. We do correlation calculation using both the sub-linear algorithm and the FFT algorithm in different vector N, and we can get the curves of the running time of the two algorithms.

We can see a giant advantage of the sub-linear time in running time complexity. The running time of the FFT algorithm is approximately 100 times than that of the sub-linear algorithm, which proves the potential value of this algorithm.

For the anti-error analysis, we do a lot of repeating correlation experiments in a certain SNR of the code and count the calculating accuracy of this SNR. We can get the curve of the calculating accuracy with SNR changing in the following Fig. 2:

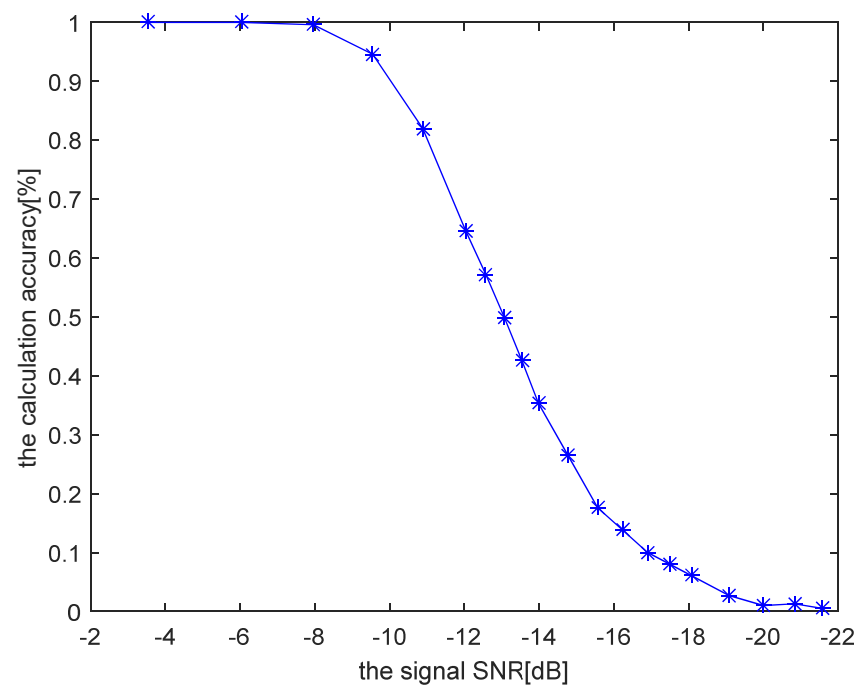

Fig. 2 the curve of the calculating accuracy with SNR changing

We can see that it has a certain performance of anti-error. And after the SNR falls down to -22dB, the accuracy decreases to its minimum.

\section{The Application of the Sub-linear Algorithm in a Passive Radar System}

In a passive radar system, in order to find the time delay and Doppler frequency offset to track the position and velocity of the aircraft, we have to do cross ambiguity function of the reflected signal from the aircraft and the reference signal by using broadcast satellite television signal as the 
emitter source. This paper applies the new algorithm to the calculation of the cross ambiguity function. The aircraft is a civil aircraft. The sample frequency of the signal is $49.107 \mathrm{MHz}$ and the distance between the aircraft and the radar is approximately $3 \mathrm{~km}$. And we can find the time delay-frequency shift curve in the following Fig. 3:

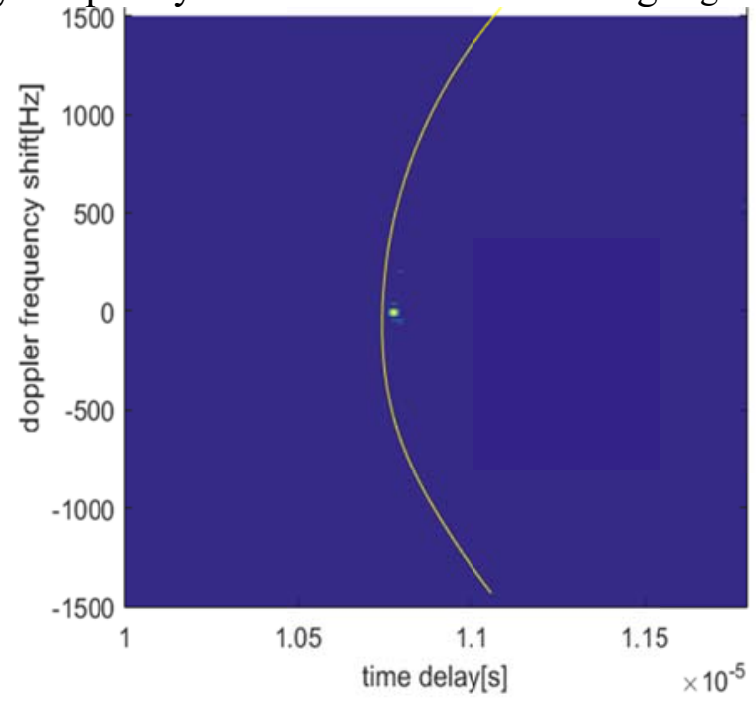

Fig. 3 (a) the FFT algorithm

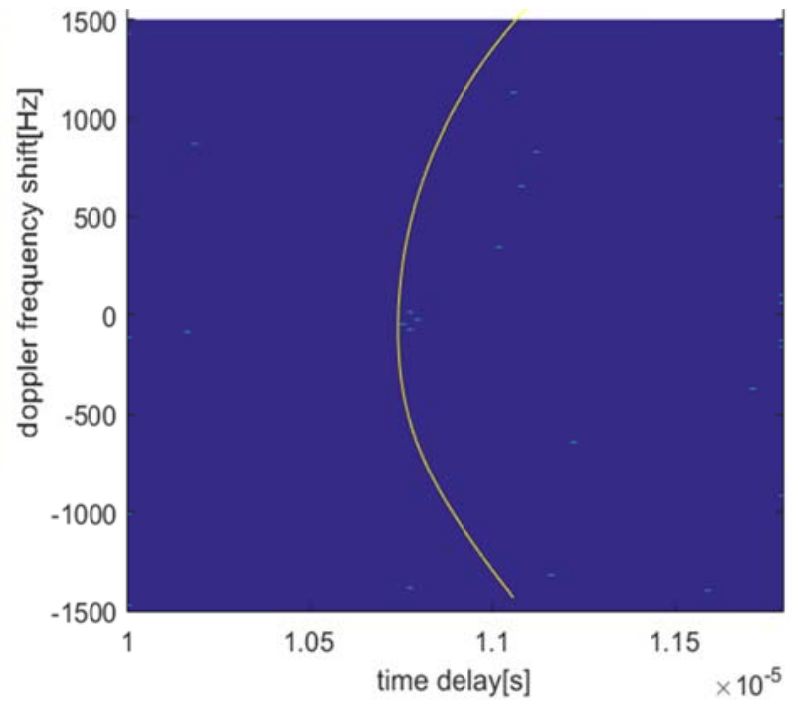

Fig. 3 (b) the sub-linear algorithm

We can see that the yellow curve is the calculated result of time delay-frequency shift from ADS-B data, which is the reference curve. The spots in the figure are the valid data we calculated from the cross ambiguity function. From the Fig. 3 (a) and Fig. 3 (b) we can see that the performance of anti-error of the sub-linear algorithm is worse than that of FFT algorithm, but in some circumstances where the SNR is higher it can get the accurate results. By adjusting the parameters the accuracy of the algorithm will rise but the running time will increase, too.

\section{Summary}

This paper applies a sub-linear time complexity correlation algorithm proposed in [1] to a passive radar system, which reduces the time complexity to $\mathrm{O}\left((\mathrm{N} \log \mathrm{N})^{2 / 3}\right)$. This paper also does experimental analysis on this sub-linear algorithm in the aspects of parameter properties, time complexity and anti-error. From the experimental results, the running time of the algorithm is much lower than the FFT algorithm and it has a certain performance of anti-error, which means it has a great advantage when the signal SNR is high. But when the SNR is lower, the accuracy will decrease too. This paper proves experimentally that this sub-linear algorithm has a great value in practical applications.

\section{References}

[1] Andoni, Alexandr, Haitham Hassanieh, Piotr Indyk, and Dina Katabi. "Shift Finding in Sub-Linear Time." Proceedings of the Twenty-Fourth Annual ACM-SIAM Symposium on Discrete Algorithms (January 6, 2013): 457- 465.

[2] H. Hassanieh, P. Indyk, D. Katabi, et al, "Simple and Practical Algorithm for Sparse Fourier Transform," SODA, 2012.

[3] H. Hassanieh, F. Adib, D. Katabi, et al, "Faster GPS via the Sparse Fourier Transform," ACM MobiCom, August 2012.

[4] H. Hassanieh, P. Indyk, D. Katabi, et al, "Nearly Optimal Sparse Fourier transform," STOC, 2012.

[5] P. Indyk, M .Kapralov, "Sample-Optimal Sparse Fourier Transform in Any Constant Dimension," FOCS, October 2014. 
[6] A. Amir, M.Lewenstein, and E. Porat. Faster algorithms for string matching k mismatches J. Algorithms, 50 (2): 257-275, 2004.

[7] T. Batu, F. Ergun, J. Kilian, A. Magen, S. Raskhodnikova, R. Rubinfeld, and R. Sami. A sublinear algorithm for weakly approximating edit dis- tance. Pages 316-324, 2003.

[8] A. Andoni and H. L. Nguyen. Near-optimal sublinear time algorithms for ulam distance. SODA, pages 76-86, 2010.

[9] M. J. Fischer and M. S. Paterson. String matching and other products Complexity of Computation, SIAM-AMS Proceedings, 7: 113-125, 1974.

[10]G.M. Landau and U. Vishkin. Fast parallel and serial approximate string matching J. Algorithms, 10 (2):157169, 1989.

[11]A. Amir, M. Lewenstein, and E. Porat. Faster algorithms for string matching with k mismatches J. Algorithms, 50 (2):257-275, 2004.

[12]RTCA, DO-260A Minimum Operational Performance Standards for $1090 \mathrm{MHz}$ Automatic Dependent Surveillance-Broadcast (ADS-B) and Traffic Information Services (TIS-B), FAA, 2003. 